\title{
Desfechos em Pacientes com Fenômeno de No-Reflow Coronário e a Relação entre a Molécula-1 de Lesão Renal e o Fenômeno de No-Reflow Coronário
}

\author{
Outcomes in Coronary No-Reflow Phenomenon Patients and the Relationship between Kidney Injury Molecule-1 \\ and Coronary No-Reflow Phenomenon
}

Mustafa Ahmet Huyut ${ }^{1}$ e Aylin Hatice Yamac ${ }^{2}$

Yeni Yuzyil University - Cardiology, ${ }^{1}$ Istanbul - Turquia

Bezmialem Vakif University - Cardiology, ${ }^{2}$ Istanbul - Turquia

Resumo

Fundamento: $\mathrm{O}$ fenômeno de no-reflow coronário (CNP, do inglês Coronary no-reflow phenomenon) está associado a um risco aumentado de eventos cardiovasculares adversos maiores (ECAM).

Objetivo: Este estudo teve como objetivo avaliar a relação entre os níveis séricos da Molécula-1 de lesão renal (KIM-1) e o CNP em pacientes com infarto agudo do miocárdio com supradesnivelamento do segmento ST (IAMCSST).

Métodos: Este estudo incluiu um total de 160 pacientes (113 homens e 47 mulheres; média de idade: 61,65 \pm 12,14 anos) com diagnóstico de IAMCSST. Os pacientes foram divididos em dois grupos, o grupo reflow $(\mathrm{GR})(\mathrm{n}=140)$ e o grupo no-reflow (GNR) $(n=20)$. Os pacientes foram acompanhados durante um ano. Um valor de $p<0,05$ foi considerado significativo.

Resultados: O CNP foi observado em 12,50\% dos pacientes. O nível de KIM-1 sérico foi significativamente maior no GNR do que no GR $(20,26 \pm 7,32$ vs. 13,45 $\pm 6,40, p<0,001)$. O índice de massa corporal (IMC) foi significativamente maior no GNR do que no GR $(29,41(28,48-31,23)$ vs. 27,56 $(25,44-31,03)$, $p=0,047)$. A frequência cardíaca (FC) foi significativamente menor no GNR do que no GR $(61,6 \pm 8,04$ vs. $80,37 \pm 14,61, p<0,001)$. O escore do European System for Cardiac Operative Risk Evaluation II (EuroSCORE II) foi significativamente maior no GNR do que no GR (3,06 $\pm 2,22$ vs. $2,36 \pm 2,85, p=0,016)$. A incidência de AVC foi significativamente maior no GNR do que no GR (15\% vs. 2,90\%, $p=0,013)$. O nível basal de KIM-1 (OR = 1,19, IC 95\%: 1,07-1,34, p=0,002) e HR (OR = 0,784, IC 95\%: 0,69$0,88, \mathrm{p}<0,001$ ) foram os preditores independentes de CNP.

Conclusão: Em conclusão, os níveis séricos basais de KIM-1 e a FC mais baixa estão independentemente associados com CNP em pacientes com IAMCSST, e o acidente vascular cerebral foi significativamente maior no GNR em um ano de seguimento. (Arq Bras Cardiol. 2021; 116(2):238-247)

Palavras-chave: Doenças Cardiovasculares; Infarto do Miocárdio; Acidente Vascular Cerebral; Intervenção Coronária Percutânea; Trombose Coronária; Frequência Cardíaca.

\begin{abstract}
Background: Coronary no-reflow phenomenon (CNP) is associated with an increased risk of major cardiovascular adverse events (MACE).

Objective: This study aimed to evaluate the relationship between serum Kidney Injury Molecule-1 (KIM-1) levels and CNP in patients with acute ST-segment elevation myocardial infarction (STEMI).

Methods: This study included a total of 160 patients (113 males and 47 females; mean age: 61.65 \pm 12.14 years) who were diagnosed with STEMI. The patients were divided into two groups, the reflow group (RG) $(n=140)$ and the no-reflow group (NRG) ( $n=20)$. Patients were followed during one year. A p-value of $<0.05$ was considered significant.

Results: CNP was observed in $12.50 \%$ of the patients. Serum KIM-1 was significantly higher in the NRG than in the RG (20.26 $\pm 7,32$ vs. 13.45 $\pm 6.40, p<0.001)$. Body mass index (BMI) was significantly higher in the NRG than in the RG (29.41 (28.48-31.23) vs. 27.56 (25.4431.03), $p=0.047)$. Heart rate (HR) was significantly lower in the NRG than in the RG $(61.6 \pm 8.04$ vs. $80.37 \pm 14.61, p<0.001)$. The European

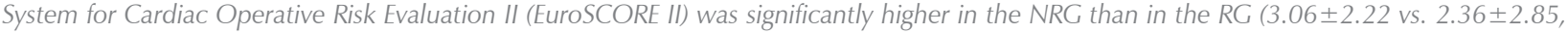

Correspondência: Mustafa Ahmet Huyut •

Yeni Yuzyil University - Cardiology - Merkez Mahallesi, Çukurçeşme Caddesi, No:51 34245 Gaziosmanpaşa / Istanbul Istanbul 34245 - Turquia E-mail: ahuyut@yahoo.com

Artigo recebido em 27/09/2019, revisado em 08/12/2019, aceito em 22/01/2020

DOI: https://doi.org/10.36660/abc.20190656 
$p=0.016)$. The incidence of stroke was significantly higher in the NRG than in the RG (15\% vs. 2.90\%, $p=0.013)$. The baseline KIM-1 level $(O R=1.19,95 \% \mathrm{Cl}: 1.07$ to $1.34, p=0.002)$ and $\mathrm{HR}(\mathrm{OR}=0.784,95 \% \mathrm{Cl}: 0.69$ to $0.88, p<0.001)$ were the independent predictors of CNP.

Conclusion: In conclusion, baseline serum KIM-1 concentrations and lower HR are independently associated with CNP in STEMI patients and the incidence of stroke was significantly higher in the NRG in the one-year follow-up. (Arq Bras Cardiol. 2021; 116(2):238-247)

Keywords: Cardiovascular Diseassses; Myocardial Infarction Stroke; Percutaneous Coronary Intervention; Coronary Thrombosis; Heart Rate.

Full texts in English - http://www.arquivosonline.com.br

\section{Introdução}

O fenômeno de no-reflow coronário (CNP, do inglês coronary no-reflow phenomenon) foi definido como falta de perfusão miocárdica apesar da abertura do vaso coronariano no contexto da intervenção coronária percutânea primária (ICP). ${ }^{1}$ Geralmente, o CNP angiográfico é definido como a presença de Thrombolysis In Myocardial Infarction (TIMI) 0-I, que se refere à perda súbita de fluxo epicárdico após dilatação com balão ou colocação de um stent sem a presença de dissecção, obstrução mecânica, estenose residual significativa, espasmo ou trombo do vaso coronário. ${ }^{2}$ Os mecanismos subjacentes do CNP são inflamação, microembolização aterotrombótica, ativação de neutrófilos e plaquetas, que causam a liberação de radicais livres de oxigênio, enzimas proteolíticas e mediadores pró-inflamatórios que podem causar dano tecidual e endotelial, particularmente em miócitos gravemente danificados. ${ }^{3}$ Biomarcadores de lesão tubular renal, como KIM-1, têm sido associados à incidência e progressão de lesão renal aguda (LRA) e doença renal crônica (DRC). ${ }^{4}$ A KIM-1 é uma proteína transmembrana do tipo 1 e é expressa na membrana apical do túbulo proximal de acordo com a lesão. ${ }^{5}$ A LRA e a DRC estão fortemente associadas à doença cardiovascular (DCV), e a LRA foi relatada como associada com eventos cardiovasculares. ${ }^{6} \mathrm{~A}$ KIM-1 atua como uma molécula pró-inflamatória e tem funções quimioatrativa e de adesão celular. ${ }^{4} \mathrm{~A}$ estrutura da KIM-1 sugere que ela pode estar envolvida nas interações de adesão de superfície. ${ }^{4-6}$ As citocinas pró-inflamatórias contribuem para a inflamação ao aumentar e estimular as células inflamatórias e a resposta inflamatória. ${ }^{4}$ A KIM-1 também tem relação espacial com células T inflamatórias. ${ }^{4}$ A KIM-1 demonstrou interagir com a proliferação de células $\mathrm{T}$, além de interagir com outras proteínas pró-inflamatórias. ${ }^{4}$ Além disso, as células T têm sido implicadas na fisiopatologia da lesão pós-isquêmica do endotélio. ${ }^{4}$ É possível que a KIM-1 desempenhe um papel importante nas células epiteliais sobreviventes para sofrer diferenciação, migração, proliferação e restauração da integridade morfológica e funcional do epitélio. No entanto, a KIM-1 está associada à fibrose e inflamação. ${ }^{4}$ Nossa hipótese é que a expressão de KIM-1 é induzida no IAMCSST e está relacionada ao CNP e ao dano endotelial devido à resposta pró-inflamatória. A associação entre os níveis da proteína KIM1 e o CNP ainda não foi abordada na literatura. Compreender quais vias biológicas e marcadores estão associados ao CNP pode permitir o desenho de estudos futuros para explorar a ligação mecanicista entre essas vias e avaliar a eficácia das intervenções destinadas a reduzir a carga de DCV nesses pacientes. Além disso, o objetivo deste estudo foi avaliar a relação entre os níveis séricos da proteína KIM-1 e o CNP em pacientes com IAMCSST agudo.

\section{Métodos}

Este estudo foi conduzido prospectivamente entre maio de 2016 e maio de 2018 no Bezmialem Vakif University Hospital. Para este estudo de centro único, incluímos 346 pacientes entre 18 e 90 anos que foram diagnosticados com IAMCSST e submetidos a ICP primária no período de 6 horas após o início dos sintomas. Todos os pacientes com IAMCSST foram encaminhados ao laboratório de cateterismo para ICP primária $(\mathrm{n}=346)$. Foram excluídos deste estudo pacientes com cirurgia de revascularização do miocárdio (CRM), choque cardiogênico, edema pulmonar com classe Killip $\geq 2$, trombose de stent, aqueles submetidos a aspiração de trombo no evento índice, pacientes com doença infecciosa ou neoplásica aguda ou crônica, doença renal crônica moderada a grave e doença hepática crônica $(n=186)$. De acordo com os resultados finais das características angiográficas do fluxo TIMI da artéria tratada, um total de 20 pacientes com CNP angiograficamente comprovado foram incluídos no GNR, sendo incluídos 140 pacientes no GR. Todos os pacientes receberam tratamento com $300 \mathrm{mg}$ de ácido acetilsalicílico e uma dose de ataque de clopidogrel (600 mg) e heparina NF (100 mg/kg) antes da ICP. Todos os participantes deram consentimento informado por escrito antes da participação e o estudo foi aprovado pelo comitê de ética local. Além disso, o estudo foi conduzido de acordo com as disposições da Declaração de Helsinque.

\section{Análises bioquímicas}

Amostras de sangue venoso foram coletadas da veia antecubital imediatamente após a admissão hospitalar antes da ICP. Eletrocardiogramas de 12 derivações foram obtidos no início do estudo e a frequência cardíaca (FC) foi anotada. O IMC foi calculado pela fórmula: peso $(\mathrm{kg}) /$ altura $^{2}\left(m^{2}\right)$. A taxa de filtração glomerular estimada (TFGe) de cada paciente foi calculada utilizando a equação do Chronic Kidney Disease Epidemiology Collaboration. Análises químicas de rotina do sangue, os parâmetros lipídicos e a troponina-I foram medidos em um autoanalisador padrão. O hemograma foi medido com um autoanalisador Sysmex K-1000 (Block Scientific, Bohemia, NY, EUA). As amostras foram centrifugadas a $3000 \mathrm{rpm}$ durante $10 \mathrm{~min}$, e o sobrenadante e o soro separados das amostras. Em seguida, foram congelados a $-80^{\circ} \mathrm{C}$ até análise posterior. Os níveis séricos de KIM-1 (ng/mL) foram medidos com o kit ELISA de ensaio imunoenzimático, comercialmente disponível (Human KIM-1 ELISA kit, Elabscience Biotech Co., Ltd, Catalog no: E-EL-H0186, Wuhan, China) ${ }^{4}$ Os coeficientes de variação inter e intra-ensaio da análise do kit KIM-1 para o ensaio foi inferior a $10 \%$, e a sensibilidade foi de $0,10 \mathrm{ng} / \mathrm{mL}$. 


\section{Diagnóstico de infarto agudo do miocárdio com elevação do segmento ST}

O diagnóstico de IAMCSST foi feito na presença das seguintes características com base nas definições de diretrizes de prática clínica: dor típica no tórax com duração superior a 30 minutos, novo início ou supostamente novo supradesnivelamento do segmento ST em duas ou mais derivações contíguas com elevação do segmento $S T \geq 2,5 \mathrm{~mm}$ em homens $<40$ anos, $\geq 2 \mathrm{~mm}$ em homens $\geq 40$ anos, ou $\geq 1,5 \mathrm{~mm}$ em mulheres nas derivações $\mathrm{V} 2-\mathrm{V} 3 \mathrm{e} / \mathrm{ou} \geq 1 \mathrm{~mm}$ nas outras derivações (na ausência de hipertrofia ventricular esquerda ou bloqueio de ramo esquerdo). Em pacientes com IM inferior registrado nas derivações precordiais direitas (elevação do segmento ST em V3R e V4R), foi considerado um infarto concomitante do ventrículo direito. Da mesma forma, o infradesnivelamento do segmento ST nas derivações V1-V3 e onda T positiva (elevação equivalente do segmento ST); além disso, a elevação concomitante do segmento $S T \geq 0,5$ $\mathrm{mm}$ registrada nas derivações $\mathrm{V} 7 \mathrm{~V} 9$ foi considerada como um IM posterior. ${ }^{7}$

\section{Fatores de risco cardiovascular}

Após exames detalhados, o histórico médico de cada paciente foi coletado pelo mesmo investigador. Fatores de risco para doença arterial coronariana (DAC), incluindo idade, sexo, hipertensão (HT), diabetes mellitus (DM), hiperlipidemia $(\mathrm{HL})$ e tabagismo, foram registrados. Pacientes que estavam anteriormente sob terapia anti-hipertensiva ou cuja pressão arterial, medida pelo menos duas vezes, era $\geq 140 / 90 \mathrm{mmHg}$, foram considerados hipertensos. ${ }^{8}$ Pacientes que estavam anteriormente em uso de antidiabético oral e/ou em terapia com insulina ou cuja glicemia de jejum, medida pelo menos duas vezes, era $\geq 125 \mathrm{mg} / \mathrm{dL}$, foram considerados diabéticos. ${ }^{9}$ A presença de $\mathrm{HL}$ foi considerada quando uma medida de colesterol total $>200 \mathrm{mg} / \mathrm{dL}$ ou colesterol de lipoproteína de baixa densidade (LDL-C) $>100 \mathrm{mg} / \mathrm{dL}$ foi obtida ou quando o paciente fazia uso prévio de medicação hipolipemiante de acordo com a diretriz do "Adult Treatment Panel III". ${ }^{10}$ Pacientes que ainda faziam uso de produtos de tabaco na admissão no serviço de emergência e que haviam parado de fumar no último mês foram considerados tabagistas.

\section{Angiografia coronária}

Os procedimentos de angiografia coronária foram realizados com equipamento de angiografia Philips (Optimus 200 DCA e Integris Allura 9, Philips Medical Systems, Eindhoven, Holanda) por via femoral. A angiografia coronária e a ICP foram realizadas de acordo com a prática clínica padrão com meio de contraste iso-osmolar não iônico (iodixanol, Visipaque 320mg/100mL; GE Healthcare, Cork, Irlanda). Foi realizada a ICP primária da artéria relacionada ao infarto. As imagens angiográficas foram registradas a uma taxa de pelo menos 80 quadros de imagem e com gravação a uma taxa de 25 quadros por segundo. Pelo menos dois cardiologistas examinaram os registros de exames anatômicos coronários offline. A velocidade do fluxo sanguíneo coronário foi determinada pelo número quantitativo de contagem de quadros, conforme descrito por Gibson et al. ${ }^{11} \mathrm{O}$ CNP foi definido angiograficamente como graus de fluxo TIMI pós$\mathrm{ICP} \leq 1$, sem a presença de dissecção, obstrução mecânica, ou estenose significativa. ${ }^{1}$ Os pacientes com CNP receberam inibidores da glicoproteína IIb/IIla intracoronários (IC) (Gp-IIb / IIla inh.) ou adenosina IC ou epinefrina IC para o tratamento de CNP, respectivamente. Após o procedimento, todos os pacientes foram submetidos a hidratação intravenosa (IV) com solução salina isotônica por pelo menos 12 horas.

\section{Ecocardiografia transtorácica}

Cada paciente foi submetido a um exame ecocardiográfico transtorácico com um transdutor de 3,5 MHz (Vivid 7 GE Medical System, Horten, Noruega) pelos mesmos investigadores antes da alta hospitalar. Os exames e medidas foram realizados de acordo com as recomendações da American Echocardiography Unit. O método de Simpson foi utilizado para calcular a fração de ejeção do ventrículo esquerdo (FEVE).

\section{Seguimento}

As informações de seguimento foram obtidas por registros hospitalares e dados de visitas dos pacientes ao hospital, 1, 3, 6 e 12 meses pelos mesmos investigadores. Os desfechos deste estudo, ECAM incluindo mortalidade por todas as causas, morte cardiovascular, acidente vascular cerebral, reinfarto do miocárdio, foram obtidos de registros hospitalares e certificados de óbito, ou contato telefônico com parentes dos pacientes.

\section{Análise estatística}

A análise dos dados foi realizada com o pacote de software estatístico SPSS versão 24.0 (SPSS Inc., Chicago, IL, EUA). O teste de Kolmogorov-Smirnov foi utilizado para controlar as distribuições das variáveis. O teste $t$ de Student para duas amostras independentes foi usado para dados normalmente distribuídos e relatados como média e desvio padrão, e o teste $U$ de Mann-Whitney foi utilizado para comparar dados não-normalmente distribuídos e relatados como mediana e percentis 25 e 75. Os dados categóricos foram comparados pelo teste Qui-quadrado. As correlações entre as variáveis foram realizadas através da análise de correlação de postos de Spearman. O método de Kaplan-Meier foi utilizado para estimar as taxas de sobrevida livre de eventos. A análise da curva de característica de operação do receptor (ROC) foi realizada para determinar o valor preditivo de KIM-1 para CNP. A análise de regressão logística foi realizada para avaliar os preditores do CNP. Foi realizada análise de regressão logística univariada, e as variáveis que se mostraram estatisticamente significativas $(p<0,1)$ foram avaliadas com a análise de regressão logística multivariada. Um valor de P bicaudal $<0,05$ foi considerado significativo.

\section{Resultados}

Este estudo incluiu um total de 160 pacientes (113 homens e 47 mulheres; média de idade: 61,65 \pm 12,14 anos) com diagnóstico de CNP. O CNP foi observado em $12,50 \%$ da população estudada. Os achados demográficos e 
medicamentos entre os grupos são descritos na Tabela 1. Em relação aos fatores de risco cardiovascular, o IMC $\left(\mathrm{kg} / \mathrm{m}^{2}\right)$ foi significativamente maior no GNR do que no GR $(29,41$ (28,48$31,23)$ vs. $27,56(25,44-31,03), p=0,047)$. As características laboratoriais basais dos pacientes são descritas na Tabela 2. O nível de KIM-1 foi significativamente maior no GNR do que no GR $(20,26 \pm 7,32$ vs. $13,45 \pm 6,40$, $p<0,001)$, a FC foi significativamente menor no GNR do que no GR $(61,60$ $\pm 8,04$ vs. $80,37 \pm 14,61, p<0,001)$ e o EuroSCORE II foi significativamente maior no GNR do que no GR (3,06 \pm 2,22 vs. 2,36 $\pm 2,85, p=0,016$ ). Em 4 pacientes, o CNP foi resolvido com inibidores $\mathrm{Gp}$-IIb/IIla IC, em 8 pacientes, o CNP foi resolvido com inibidores Gp-IIb/IIla IC mais adenosina IC e em 5 pacientes o CNP foi resolvido com inibidores Gp-Illb/ IIla IC mais adenosina IC e epinefrina IC (Tabela 1). Em 17 pacientes o CNP foi resolvido e eles foram incluídos no GR. O CNP persistiu em 20 pacientes e eles foram incluídos no GNR.

Os achados do seguimento clínico, incluindo mortalidade por todas as causas, morte cardiovascular, acidente vascular cerebral, infarto do miocárdio e ECAM foram descritos na Tabela 3. O acidente vascular cerebral foi significativamente maior no GNR do que no GR ( $15 \%$ vs. 2,9\%, p=0,013). Não houve diferença entre os dois grupos em relação a outros achados demográficos ou clínicos. As curvas de Kaplan-Meier para as taxas de AVC e ECAM são descritas nas Figuras 1 e 2. Idade, TFGe, escore de Mehran, FEVE e hs-PCR foram significativamente associados ao EuroSCORE II $(p<0,05)$ (Tabela 4). A análise de regressão logística condicional forward demonstrou que KIM-1 (OR = 1,199, IC 95\%: 1,07-1,343, $p=0,002)$ e HR (OR = 0,784, IC 95\%: 0,696-0,883, $p<0,001)$ foram os preditores independentes de CNP em pacientes com IAMCSST (Tabela 5). Na análise ROC, os valores de KIM-1 sérico acima de 21,53 ng/mL previram a presença de CNP com $85 \%$ de sensibilidade e $93,6 \%$ de especificidade. A área sob a curva foi de 0,80 (IC 95\%: 0,653-0,946, p<0,001) (Figura 3).

\section{Discussão}

O principal achado deste estudo foi que o aumento dos níveis de KIM-1 e a redução da FC foram os dois determinantes do CNP. Mostramos que os valores de KIM-1 sérica acima de 21,53 ng/mL sugerem a presença de CNP; portanto, níveis elevados de KIM-1 sérica podem ser utilizados como

Tabela 1 - Características basais e medicamentos dos pacientes

\begin{tabular}{|c|c|c|c|}
\hline Variável, n(\%) & GNR $n=20(12,5)$ & GR n=140 $(87,5)$ & Valor de $\mathrm{p}$ \\
\hline Idade, a & $64,35 \pm 14,03$ & $61 \pm 11,86$ & 0,291 \\
\hline Sexo masculino, n (\%) & $11(55,00)$ & $102(72,90)$ & 0,101 \\
\hline IMC $\left(\mathrm{kg} / \mathrm{m}^{2}\right)$ & $29,41(28,48-31,23)$ & $27,56(25,44-31,03)$ & 0,047 \\
\hline HT, n (\%) & $15(75)$ & $79(56,40)$ & 0,115 \\
\hline $\mathrm{DM}, \mathrm{n}(\%)$ & $7(35)$ & $50(35,70)$ & 0,950 \\
\hline $\mathrm{HL}, \mathrm{n}(\%)$ & $6(30)$ & $62(44,30)$ & 0,227 \\
\hline Fumante, n (\%) & $11(55)$ & $90(64,30)$ & 0,421 \\
\hline Histórico familiar, n (\%) & $6(30)$ & $54(38,60)$ & 0,459 \\
\hline DAP, n (\%) & $3(15)$ & $11(7,90)$ & 0,290 \\
\hline DPOC, n (\%) & $3(15)$ & $26(18,60)$ & 0,698 \\
\hline FEVE (\%) & $51,25 \pm 6,72$ & $52,01 \pm 7,49$ & 0,561 \\
\hline \multicolumn{4}{|l|}{ Medicamentos n (\%) } \\
\hline Inib. ECA & $14(70)$ & $75(53,60)$ & 0,167 \\
\hline BRA & $5(25)$ & $44(31,40)$ & 0,560 \\
\hline B Bloqueador & $19(95)$ & $133(95)$ & 1 \\
\hline $\mathrm{BCC}$ & $6(30)$ & $34(24,30)$ & 0,581 \\
\hline Estatina & $20(100)$ & $124(88,60)$ & 0,111 \\
\hline Nitrato & $9(45)$ & $49(35)$ & 0,384 \\
\hline ADO & $7(35)$ & $48(34,30)$ & 0,950 \\
\hline Inib. Gp-IIb/IIla IC & $20(100)$ & $17(12,10)$ & $<0,001$ \\
\hline Adenosina IC & $20(100)$ & $13(9,3)$ & $<0,001$ \\
\hline Epinefrina IC & $20(100)$ & $5(3,6)$ & $<0,001$ \\
\hline
\end{tabular}




\begin{tabular}{|c|c|c|c|}
\hline Variável, n(\%) & GNR $n=20(12,5)$ & GR n=140 $(87,5)$ & Valor de $p$ \\
\hline $\mathrm{KIM}-1 \mathrm{ng} / \mathrm{mL}$ & $20,26 \pm 7,32$ & $13,45 \pm 6,40$ & $<0,001$ \\
\hline Glicose, mg/dl & $134,25 \pm 65,06$ & $136,73 \pm 61,27$ & 0,689 \\
\hline Ácido úrico, mg/dl & $5,63 \pm 1,51$ & $5,73 \pm 1,73$ & 0,883 \\
\hline TFGe $\left(\mathrm{mL} / \mathrm{min}\right.$ por $\left.1,73 \mathrm{~m}^{2}\right)$ & $75,54 \pm 22,63$ & $82,3 \pm 21,47$ & 0,154 \\
\hline Escore de Mehran & $5(2-8)$ & $3,5(2-6,75)$ & 0,145 \\
\hline Desenvolvimento NIC, n (\%) & $1(5)$ & $14(10)$ & 0,473 \\
\hline $\mathrm{HC}, 10^{3} / \mathrm{uL}$ & $9,86 \pm 4,32$ & $9,45 \pm 3,30$ & 0,796 \\
\hline HTC,\% & $40,07 \pm 3,44$ & $40,36 \pm 4,66$ & 0,678 \\
\hline Plaquetas, $10^{3} / \mathrm{uL}$ & $231,60 \pm 62,13$ & $238,83 \pm 73,29$ & 0,520 \\
\hline Internação hospitalar, dias & $3,15 \pm 0,48$ & $3 \pm 1,12$ & 0,408 \\
\hline Triglicérides, (mg/dL) & $160,50 \pm 37,62$ & $155,37 \pm 57,52$ & 0,323 \\
\hline $\mathrm{HDL},(\mathrm{mg} / \mathrm{dL})$ & $39,70 \pm 5,01$ & $41,05 \pm 7,83$ & 0,938 \\
\hline LDL, (mg/dL) & $138,15 \pm 31,86$ & $123,77 \pm 33,91$ & 0,076 \\
\hline Colesterol total, (mg/dL) & $214,15 \pm 33,47$ & $200,75 \pm 38,1$ & 0,188 \\
\hline hs-PCR, (mg/dL) & $0,10(0,01-0,43)$ & $0,20(0,05-0,50)$ & 0,532 \\
\hline Pico da troponina-I (pg/mL) & $2293(432,75-13501,25)$ & $808,50(68,25-3770,50)$ & 0,220 \\
\hline Frequência cardíaca, (bpm) & $61,6 \pm 8,04$ & $80,37 \pm 14,61$ & $<0,001$ \\
\hline TSH, ulU/mL & $1,05(0,70-1,30)$ & $1,10(0,90-1,40)$ & 0,245 \\
\hline Classe NYHA & $2,45 \pm 0,51$ & $2,30 \pm 0,53$ & 0,278 \\
\hline EuroSCORE II, (\%) & $3,06 \pm 2,22$ & $2,36 \pm 2,85$ & 0,016 \\
\hline
\end{tabular}

Tabela 3 - Achados do seguimento clínico

\begin{tabular}{lccc}
\hline Variável, $\mathbf{n}(\%)$ & GNR $\mathbf{n = 2 0 ( 1 2 , 5 )}$ & GR $\mathbf{n = 1 4 0 ~ ( 8 7 , 5 )}$ & Valor de $\mathbf{p}$ \\
\hline Mortalidade por todas as causas & $2(10)$ & $21(15)$ & 0,551 \\
\hline Morte Cardiovascular & $2(10)$ & $16(11,4)$ & 0,850 \\
\hline Derrame & $3(15)$ & $4(2,9)$ & 0,013 \\
\hline Infarto do miocárdio & $2(10)$ & $17(12,1)$ & 0,782 \\
\hline ECAM & $6(30)$ & $35(25)$ & 0,682 \\
\hline
\end{tabular}

Os dados foram relatados como $n$ (\%). ECAM: Eventos cardiovasculares adversos maiores.

um biomarcador promissor de CNP. Em nosso estudo, descobrimos que o CNP foi independentemente associado às concentrações séricas basais de KIM-1 e menor FC em pacientes com IAMCSST. Que seja de nosso conhecimento, este é o primeiro relato na literatura que demonstra a relação entre as concentrações de KIM-1 e FC mais baixa com CNP. Além disso, em pacientes com IAMCSST, o CNP foi significativamente associado a resultados ruins. No seguimento clínico de um ano, os achados demonstraram que a ocorrência de AVC foi significativamente maior no grupo GNR.

Embora o mecanismo exato do CNP não seja determinado de forma consistente na literatura, existem vários mecanismos sugeridos. Esses mecanismos são relatados como disfunção microvascular pré-existente, espasmo arteriolar microvascular, tromboembolização distal devido à alta atividade plaquetária e carga trombótica, lesão de reperfusão isquêmica, edema das células do miocárdio comprimindo os vasos microvasculares. ${ }^{1-3}$ Portanto, a patogênese e mecanismos do CNP permanecem controversos.

O CNP é um marcador prognóstico significativo relacionado a desfechos cardíacos ruins em curto prazo no IAMCSST. Em relação aos dados publicados, a frequência das estimativas do CNP foi de $5 \%$ a $60 \% .{ }^{12}$ Em nosso estudo, o CNP foi observado em $12,50 \%$ da população estudada. Consistente com os dados 


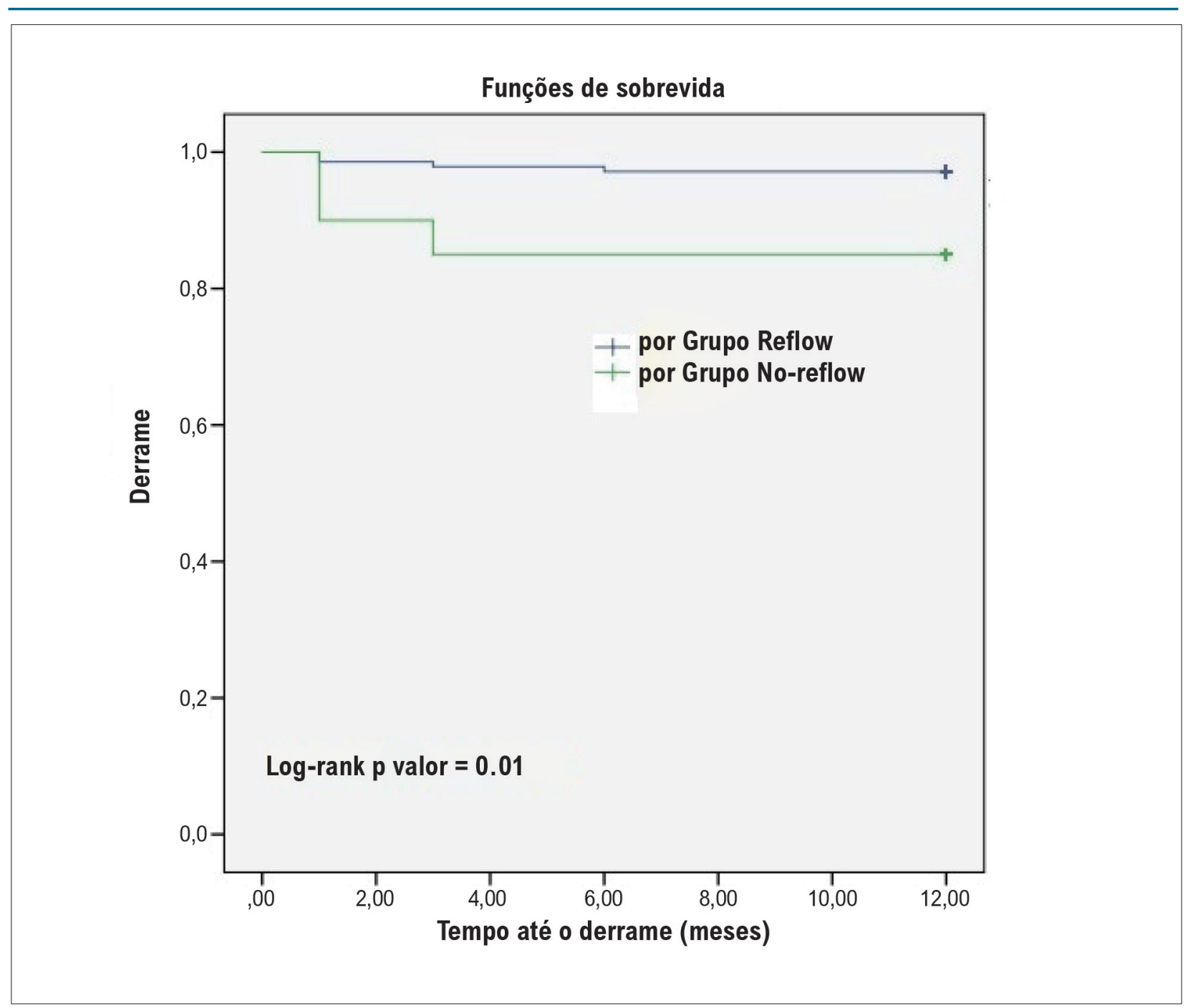

Figura 1 - Curva de Kaplan-Meier para AVC.

publicados, os pacientes com CNP apresentaram desfechos piores. ${ }^{13} \mathrm{Em}$ nosso estudo, os achados de seguimento clínico de um ano demonstraram que a ocorrência de AVC foi significativamente maior no GNR e o AVC foi associado à carga trombótica. De acordo com nosso estudo, o mecanismo associado subjacente a esse evento adverso é a ativação contínua do trombo, ainda em curso após o evento índice, e esta talvez seja a principal razão para o AVC. Embora todos os pacientes com IAMCSST tomassem medicamentos antitrombóticos regularmente, a incidência de AVC foi significativamente maior no GNR. Portanto, tais pacientes devem ser monitorados e acompanhados cuidadosamente. O IMC é a ferramenta mais amplamente utilizada para a avaliação da obesidade. ${ }^{14}$ Bakirci et al., ${ }^{15}$ descobriram que o aumento da gordura epicárdica em pacientes obesos estava associado ao fluxo coronário prejudicado em pacientes com IMCSST. ${ }^{15}$ Estudos recentes sugeriram que o CNP é mais frequentemente visto em associação com hiperglicemia e hipercolesterolemia e insuficiência renal leve a moderada. ${ }^{16}$ No entanto, a compreensão desses fatores de risco na patogênese do CNP é limitada e controversa. Em nosso estudo, descobrimos que o IMC foi significativamente maior no GNR. Pode-se comprovar que isso também está associado ao risco de acidente vascular cerebral. Assim, o cálculo do IMC pode ser um método útil para estimar os desfechos cardíacos no CNP. Além disso, diminuir o IMC pode proteger os pacientes contra o AVC. Estudos randomizados utilizaram aspiração manual de trombo e mostraram melhor perfusão microvascular e desfechos em longo prazo em comparação com pacientes controle submetidos a ICP durante o IAMCSST. ${ }^{17}$ Entretanto, o uso de aspiração de trombo pode causar acidente vascular cerebral devido à complicações com o dispositivo, e por esse motivo em nosso estudo excluímos os pacientes ( $\mathrm{n}=$ 12) do estudo nos quais utilizamos cateter de aspiração de trombo. O uso rotineiro de inibidores de plaquetas (inibidores Gp-Ilb/IIla, Abciximab, tirofiban), nicorandil, nitroprussiato e adenosina, demonstraram efeitos benéficos na perfusão miocárdica em IAMCSST. ${ }^{18-20}$ Aksu et al., ${ }^{21}$ descobriram que a epinefrina também tem um efeito benéfico sobre o CNP. ${ }^{21}$ A epinefrina causa um potente efeito vasodilatador coronário 


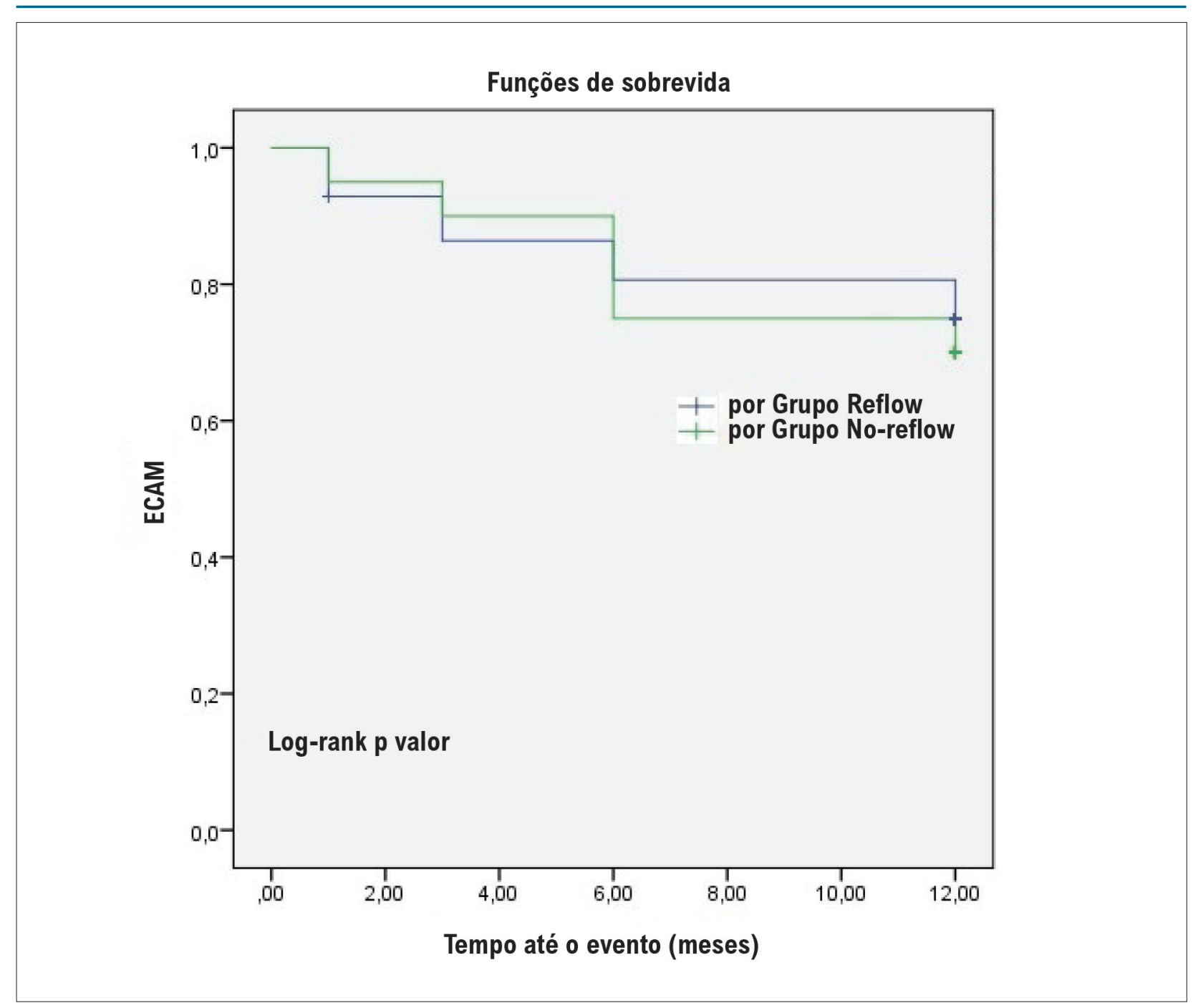

Figura 2 - Curva de Kaplan-Meier para ECAM.

Tabela 4 - Correlações entre EuroSCORE II com parâmetros clínicos

\begin{tabular}{lll}
\hline Variável & r-valor & p-valor \\
\hline Idade & 0,64 & $<0,001$ \\
\hline TFGe & $-0,64$ & $<0,001$ \\
\hline Escore de Mehran & 0,77 & $<0,001$ \\
\hline FEVE, $(\%)$ & $-0,70$ & $<0,001$ \\
\hline hs-PCR (mg/dL) & 0,24 & 0,002 \\
\hline
\end{tabular}

r: coeficiente de correlação de postos de Spearman; TFGe: taxa de filtração glomerular estimada; FEVE: fração de ejeção do ventrículo esquerdo; hs-PCR: proteína $C$ reativa de alta sensibilidade.

através da ativação do receptor beta-2, que faz a mediação da vasodilatação da circulação arteriolar. Além disso, tem efeitos cronotrópicos e inotrópicos no coração. ${ }^{22}$ A epinefrina IC pode restaurar a pressão arterial normotensa nesses pacientes, uma vez que este agente estimula os receptores vasoconstritores
Tabela 5 - Preditores independentes de CNP em IAMCSST

\begin{tabular}{llll}
\hline Variável & OR & IC95\% & Valor de $\mathbf{p}$ \\
\hline KIM-1 & 1,199 & $1,07-1,343$ & 0,002 \\
\hline FC & 0,784 & $0,696-0,883$ & $<0,001$ \\
\hline
\end{tabular}

KIM-1: molécula-1 de lesão renal; FC: frequência cardíaca; bpm: batimento por minuto; OR: Odds ratio; IC: intervalo de confiança.

alfa. ${ }^{21}$ Skelding et al.,${ }^{24}$ descobriram que o aumento do fluxo coronariano devido à correção da hipotensão pode ser o outro efeito benéfico potencial da epinefrina. ${ }^{22} \mathrm{Em}$ nosso estudo, descobrimos que a FC mais baixa estava independentemente associada ao CNP. Se a microcirculação for lenta, o CNP ocorrerá, e sugerimos que a FC mais baixa pode ser utilizada como um indicador de CNP. Além disso, os operadores devem estar cientes da FC do paciente, e um paciente com FC menor deve ser considerado um candidato ao CNP antes de iniciar a ICP. Apesar dos resultados encorajadores de nosso estudo, os 


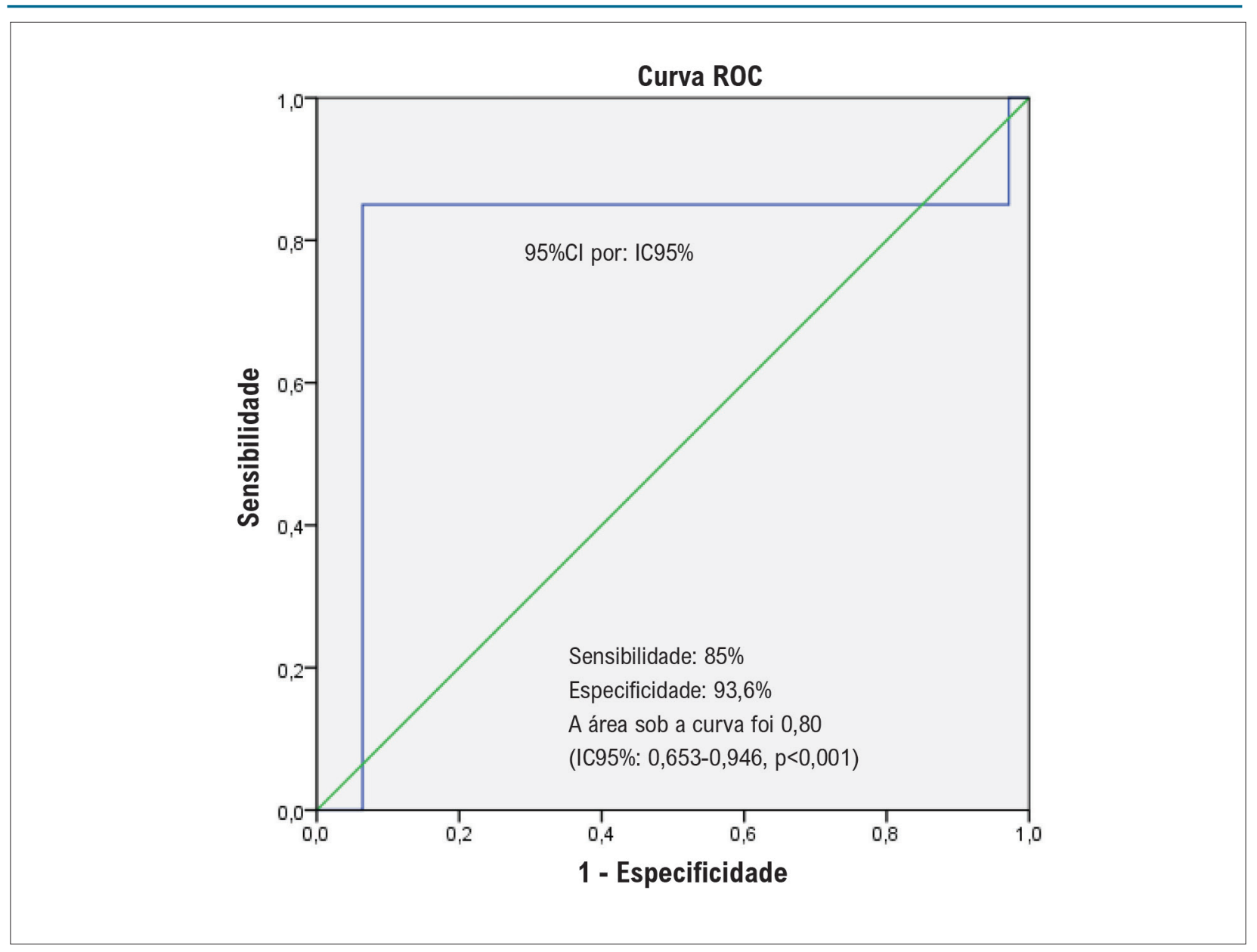

Figura 3 - Curva de análise ROC para a especificidade e sensibilidade da KIM-1 sérica.

achados de FC mais baixos devem ser explicados por grandes estudos randomizados.

O EuroSCORE II nos mostra a fragilidade e enfermidade do paciente. ${ }^{23}$ Gül et al., ${ }^{24}$ descobriram que pacientes com IAMCSST com EuroSCORE II maior apresentavam CNP significativamente maior. ${ }^{24}$ Neste estudo, no GNR, calculamos um EuroSCORE II significativamente maior, o que é consistente com a literatura. Além disso, descobrimos que idade, TFGe, escore de Mehran, FEVE e hs-PCR foram significativamente associados ao EuroSCORE II.

A KIM-1 é eliminada no sangue e na urina e serve como um indicador de diagnóstico precoce e sensível de lesão tubular proximal em humanos quando comparada a qualquer um dos marcadores de diagnóstico convencionais, por exemplo, creatinina sérica e cistatina $\mathrm{C}$ ou proteinúria. ${ }^{4} \mathrm{Em}$ condições normais, níveis muito baixos de KIM-1 são expressos nos túbulos renais proximais. Entretanto, no rim isquêmico, a expressão de KIM-1 está significativamente aumentada. ${ }^{25} \mathrm{~A}$ KIM-1 mostrou interação com a proliferação de células T. Estudos publicados sugeriram que a KIM-1 também interage com outras proteínas pró-inflamatórias. ${ }^{4-25}$ Além disso, as células T têm sido implicadas na fisiopatologia da lesão pósisquêmica do endotélio. ${ }^{4} \mathrm{~A}$ estrutura proteica da KIM-1 atua como um molécula de adesão para a superfície celular. ${ }^{25}$ Portanto, especulamos que a KIM-1 pode alterar a adesão celular e modular as interações entre a célula epitelial lesada e o conteúdo luminal, que inclui cilindros, detritos e células epiteliais viáveis que foram desalojadas do endotélio intimal e podem causar o CNP. A KIM-1 pode aumentar a mobilidade e a proliferação das células epiteliais sobreviventes. ${ }^{25}$ Macrófagos e linfócitos T são a principal fonte de várias citocinas e moléculas que interagem com as células endoteliais, o que leva a um agravamento das vias inflamatórias. Disfunção endotelial, inflamação e expressão aumentada desconhecida de agentes vasoativos, como moléculas de endotelina-1 e angiotensina, são os principais responsáveis pelos mecanismos fisiopatológicos. ${ }^{4-25} \mathrm{~A}$ inflamação desempenha um papel importante no desenvolvimento e progressão do CNP. Portanto, parece lógico combinar essas vias pró-inflamatórias para explicar os mecanismos subjacentes do CNP. A KIM-1 não somente leva à agregação de macrófagos e linfócitos $T$, mas também aumenta a secreção de citocinas oxidativas. $\mathrm{O}$ aumento de KIM-1 foi associado à inflamação sistêmica e disfunção endotelial, e foi definido como um novo marcador prognóstico baseado em inflamação na DCV. ${ }^{6}$ As principais ligações fisiopatológicas entre a KIM-1 e o CNP podem ser a adesão celular, disfunção endotelial e pró -inflamação. 


\section{Artigo Original}

Os resultados deste estudo mostram que as concentrações séricas de KIM-1 estão positivamente associadas ao CNP. Propomos que inflamação, microembolização aterotrombótica, ativação de neutrófilos e plaquetas, que causam a liberação de radicais livres de oxigênio, enzimas proteolíticas e mediadores pró-inflamatórios que podem causar dano tecidual e endotelial, particularmente em miócitos gravemente danificados durante o IAMCSST, são os mecanismos iniciais de CNP. Esses mecanismos comuns também atuam em todos os órgãos sensíveis à isquemia, especialmente nos rins e no coração. Podemos usar a KIM-1 como um marcador prognóstico precoce do CNP. No entanto, não se determinou o mecanismo exato do KIM-1 na patogênese desse fenômeno. Que seja de nosso conhecimento, este é o primeiro relato na literatura que demonstra a relação entre a KIM-1 e o CNP.

\section{Limitações}

Primeiro: embora tenhamos realizado um modelo de Cox multivariado para ajustar os fatores de confusão, um viés foi inevitável, porque este foi um estudo de centro único que incluiu um tamanho de amostra relativamente pequeno. Um estudo multicêntrico envolvendo mais pacientes poderia ter resultados e dados mais significativos. Segundo: Utilizamos apenas parâmetros angiográficos na determinação do CNP, a microcirculação não foi avaliada diretamente, seja por ecocardiografia contrastada ou por ressonância magnética, para confirmar a reperfusão adequada em nível microvascular. Esses fatores são limitações de nosso estudo.

\section{Conclusão}

Em conclusão, a inflamação desempenha um papel importante no desenvolvimento e progressão do CNP.

\section{Referências}

1. Rezkalla SH, Kloner RA. No-reflow phenomenon. Circulation. 2002 Feb 5;105(5):656-62.

2. Tasar O, Karabay AK, Oduncu V, Kirma C. Predictors and outcomes of noreflow phenomenon in patients with acute ST-segment elevation myocardial infarction undergoing primary percutaneous coronary intervention. Coron Artery Dis. 2019 Jun;30(4):270-6.

3. Yarlioglues M, Yalcinkaya D, Celik IE, Duran M. CHA2DS2VASC Score and Coronary No-Reflow Phenomenon. Angiology. 2019 May 23:3319719851698. doi: 10.1177/0003319719851698.

4. van Timmeren MM, van den Heuvel MC, Bailly V, Bakker SJ, van Goor $\mathrm{H}$, Stegeman CA. Tubular kidney injury molecule-1 (KIM-1) in human renal disease. J Pathol. 2007 Jun;212(2):209-17.

5. Go AS, Chertow GM, Fan D, McCulloch CE, Hsu CY. Chronic kidney disease and the risks of death, cardiovascular events, and hospitalization. N Engl J Med. 2004 Sep 23;351(13):1296-305. Erratum in: N Engl J Med. 2008;18(4):4.

6. Gammelager H, Christiansen CF, Johansen MB, Tønnesen E, Jespersen B, Sørensen HT. Three-year risk of cardiovascular disease among intensive care patients with acute kidney injury: A population-based cohort study. Crit Care. 2014 Oct 14;18(5):492.
Portanto, altas concentrações de KIM-1, que é definida como um marcador pró-inflamatório, podem refletir e conduzir os mecanismos subjacentes ao CNP. Além disso, as concentrações séricas basais de KIM-1 e a FC mais baixa são preditores independentes de CNP em pacientes com IAMCSST, e a incidência de AVC foi significativamente maior nesses pacientes no seguimento de um ano.

\section{Agradecimento}

Agradecemos Betul Cetintulum Huyut por suas contribuições para análises estatísticas, Hifa Gulru Caglar por suas contribuições para análises bioquímicas e todos os operadores que trabalharam no laboratório de cateterismo.

\section{Contribuição dos autores}

Concepção e desenho da pesquisa, Análise e interpretação dos dados, Análise estatística e Obtenção de financiamento: Huyut MA; Obtenção de dados, Redação do manuscrito e Revisão crítica do manuscrito quanto ao conteúdo intelectual importante: Huyut MA, Yamac AH.

\section{Potencial conflito de interesses}

Declaro não haver conflito de interesses pertinentes.

\section{Fontes de financiamento}

O presente estudo foi financiado pelo Bezmialem Vakif University, com número de financiamento 5.2016 / 17.

\section{Vinculação acadêmica}

Não há vinculação deste estudo a programas de pósgraduação.

7. Ibanez B, James S, Agewall S, Antunes MJ, Bucciarelli-Ducci C, Bueno H, et al. 2017 ESC Guidelines for the management of acute myocardial infarction in patients presenting with ST-segment elevation: the Task Force for the management of acute myocardial infarction in patients presenting with STsegment elevation of the European Society of Cardiology (ESC). Eur Heart J. 2018;39(2):119-77.

8. Armstrong C, Joint National Committee. JNC 8 Guidelines for the Management of Hypertension in Adults, Am Fam Physician 2014 Oct 1; 90(7):503-4.

9. Expert Committee on the Diagnosis and Classification of Diabetes Mellitus. Report of the expert committee on the diagnosis and classification of diabetes mellitus. Diabetes Care. 2003;26(Suppl 1): S5-20.

10. National Cholesterol Education Program (NCEP) Expert Panel on Detection, Evaluation, and Treatment of High Blood Cholesterol in Adults (Adult Treatment Panel III). Third Report of the National Cholesterol Education Program (NCEP) expert panel on detection, evaluation, and treatment of high blood cholesterol in adults (adult treatment Panel III) final report. Circulation. 2002;106:3143-421.

11. Gibson CM, Cannon CP, Daley WL, Dodge JT Jr, Alexander B Jr, Marble SJ, et al. TIMI frame count: a quantitative method of assessing coronary artery flow. Circulation 1996 Mar 1;93(5):879-88. 
12. Durante A, Camici PG. Novel insights into an "old" phenomenon: the no reflow. Int J Cardiol. 2015;187:273-80.

13. Ndrepepa G, Tiroch K, Fusaro M, Keta D, Seyfarth M, Byrne RA, et al. 5-year prognostic value of no-reflow phenomenon after percutaneous coronary intervention in patients with acute myocardial infarction. J Am Coll Cardiol. 2010 May 25;55(21):2383-9.

14. Bergman RN, Kim SP, Catalano KJ, Hsu IR, Chiu JD, Kabir M, et al. Why visceral fat is bad: mechanisms of the metabolic syndrome. Obesity (Silver Spring). 2006 Feb;14(suppl 1):16-19.

15. Bakirci EM, Degirmenci H, Duman H, Inci S, Hamur H, Buyuklu M, et al. Increased epicardial adipose tissue thickness is associated with angiographic thrombus burden in the patients with non-st-segment elevation myocardial infarction. Clin Appl Thromb Hemost. 2015 Oct;21(7):612-8.

16. Ipek G, Onuk T, Karatas MB, Gungor B, Osken A, Keskin M, et al. CHA2DS2-VASc score is a predictor of no-reflow in patients with ST-segment elevation myocardial infarction who underwent primary percutaneous intervention. Angiology. 2016 Oct;67(9):840-5.

17. Vlaar PJ, Svilaas T, van der Horst IWC, Diercks GFH, Fokkema ML, de Smet BJGL, et al. Cardiac death and reinfarction after 1 year in the thrombus aspiration during percutaneous coronary intervention in acute myocardial infarction Study (TAPAS): a 1 -year follow-up study. Lancet. 2008 Jun 7:371(9628):1915-20.

18. Danzi G, Sesana M, Capuano C, Mauri L, Centurini PB, Baglini R. Comparison in patients having primary coronary angioplasty of abciximab versus tirofiban on recovery of left ventricular function. Am J Cardiol. 2004 Jul 1;94(1):35-9.
19. Hillegass WB, Dean NA, Liao L, Rhinehart RG, Myers PR. Treatment of noreflow and impaired flow with the nitric oxide donor nitroprusside following percutaneous coronary interventions: initial human clinical experience. J Am Coll Cardiol. 2001 Apr;37(5):1335-43.

20. Amit G, Cafri C, Yaroslavtsev S, Fuchs S, Paltiel O, Abu-Ful A, et al. Intracoronary nitroprusside for the prevention of the no-reflow phenomenon after primary percutaneous coronary intervention in acute myocardial infarction. A randomized, double-blind, placebo-controlled clinical trial. Am Heart J. 2006 Nov; 152(5):887. e9-14.

21. Aksu T, Guler TE, Colak A, Baysal E, Durukan M, Sen T, et al. Intracoronary epinephrine in the treatment of refractory no-reflow after primary percutaneous coronary intervention: a retrospective study. BMC Cardiovasc Disord. 2015 Feb 19;15:10.

22. Skelding KA, Goldstein JA, Mehta L, Pica MC, O'Neill WW. Resolution of refractory no-reflow with intracoronary epinephrine. Catheter Cardiovasc Interv. 2002 Nov; 57(3):305-9.

23. Capodanno D, Dipasqua F, Marcantoni C, Ministeri M, Zanoli L, Rastell $\mathrm{S}$, et al. EuroSCORE II versus additive and logistic EuroSCORE in patients undergoing percutaneous coronary intervention. Am J Cardiol. 2013 Aug $1 ; 112(3): 323-9$.

24. Gul I, Zungur M, Aykan A, Islamli A, Yildiz B , Bilgin M. Importance of EuroSCORE-II in the Development of Acute Ischemic Heart Failure After Acute Anterior ST Elevation Myocardial Infarction. Koşuyolu Heart Journal. 2016; 19(2): 71-65.

25. Ichimura T, Hung CC, Yang SA, Stevens JL, Bonventre JV. Kidney injury molecule-1: a tissue and urinary biomarker for nephrotoxicant-induced renal injury. Am J Physiol Renal Physiol. 2004 Mar;286(3):F552-63. 